\title{
Various Numerical Methods for Singularly Perturbed Boundary Value Problems
}

\author{
Hradyesh Kumar Mishra*, Sonali saini \\ Department of Mathematics, Jaypee University of Engineering \&Technology, Madhya Pradesh, India \\ *Corresponding author: hk.mishra@juet.ac.in; sonali.saini1386@gmail.com
}

Received March 09, 2014; Revised May 08, 2014; Accepted May 09, 2014

\begin{abstract}
The numerical treatment of singular perturbation problems is currently a field in which active research is going on these days. Singular perturbation problems in which the term containing the highest order derivative is multiplied by a small parameter $\varepsilon$, occur in a number of areas of applied mathematics, science and engineering among them fluid mechanics (boundary layer problems) elasticity (edge effort in shells) and quantum mechanics. In this paper, we consider few numerical methods for singularly perturbed boundary value problems developed by numerous researchers between 2006 to 2013. A Summary of the result of some recent methods is presented and this leads to conclusion and recommendations regarding methods to use on singular perturbation problem.
\end{abstract}

Keywords: singular perturbation, ordinary differential equation, boundary layer, two-point boundary value problem, delay differential equations, integral equations

Cite This Article: Hradyesh Kumar Mishra, and Sonali saini, "Various Numerical Methods for Singularly Perturbed Boundary Value Problems." American Journal of Applied Mathematics and Statistics, vol. 2, no. 3 (2014): 129-142. doi: 10.12691/ajams-2-3-7.

\section{Introduction}

As Science \& technology develop, many practical problems, such as the mathematical boundary layer theory or approximation of solution of various problems described by differential equations involving large or small parameters have become increasingly complex and therefore require the use of asymptotic methods. However, the theory of asymptotic analysis for differential operators has mainly been developed for regular perturbations where the perturbations are subordinate to the unperturbed operator. In some problems, the perturbations occur over a very narrow region across which the dependent variable undergoes very rapid changes. These narrow regions are frequently adjacent to the boundaries of the domain of interest because a small parameter multiplies the highest derivative. Consequently, they are usually referred to as boundary layers in fluid mechanics, edge layers in solid mechanics, skin layer in electrical applications, shock layers in fluid and solid mechanics, transition points in quantum mechanics, WKB problems, the modeling of steady and unsteady viscous flow problems with large Reynolds numbers and convective heat transport problems with large peclet numbers.

In a continuation of the survey presented in $[1,2,3]$, we review methods (not all but few of them) developed by numerous researchers from 2006 to 2013 to deal with onedimensional singular perturbation problems. These problems considered include linear, non-linear reactiondiffusion, delay differential equations. The numerical techniques reviewed in this survey include finite- difference methods, spline approximation methods and computational methods for boundary value techniques.

This paper contains the following sections:

Section 2: Linear singular perturbation boundary value problems

Section 3: Non-linear singular perturbation boundary value problems

Section 4: Parameterized singular perturbation boundary value problems

Section 5: Delay differential equations

Section 6: Integral equations

Section 7: Discussion and further development.

\section{Linear Singular Perturbation Boundary Value Problems}

The basic aim of this study is to introduce and describe a patching approach based on a novel combination of the variational iterative method (VIM) [4] and adaptive cubic spline collocation scheme for the solution of a class of self-adjoint singularly perturbed second-order two-point boundary value problems that model various engineering problems. The domain of the problem is decomposed into two subintervals: the VIM is implemented in the vicinity of the boundary layer while in the outer region the resulting problem is tackled by applying an adaptive cubic spline collocation scheme (ASS), which comprises the use of mapping/transformation redistribution functions or constructed grading functions. Numerical results, computational comparisons, appropriate error measures and illustrations are provided to testify the convergence, efficiency and applicability of the method. Performance of 
this method is examined through test examples that reveal that the current approach converges to the exact solution rapidly and with $\mathcal{O}\left(h^{4}\right)$ accuracy and that the convergence is uniform across the domain. The proposed technique yields numerical solutions in very good agreement with and/or superior to existing exact and approximate solutions.

In [5], a numerical method is proposed for solving singularly perturbed turning point problems exhibiting twin boundary layers based on the reproducing kernel method (RKM). The original problem is reduced to two boundary layers problems and a regular domain problem. The regular domain problem is solved by using the RKM. Two boundary layers problems are treated by combining the method of stretching variable and the RKM. The boundary conditions at transition points are obtained by using the continuity of the approximate solution and its first derivatives at these points. Two numerical examples are provided to illustrate the effectiveness of the present method. The results compared with other methods show that the present method can provide very accurate approximate solutions.

In [6], a reliable algorithm is presented to develop approximate analytical solutions of fourth order singularly perturbed two-point boundary value problems in which the highest order derivative is multiplied by a small parameter. In this method, first the given problem is transformed into a system of two second order ODEs, with suitable boundary conditions and a zeroth-order asymptotic approximate solution of the transformed system is constructed. Then, the reduced terminal value system is solved analytically using the differential transform method. Some illustrating examples are solved and the results are compared with the exact solutions to demonstrate the accuracy and the efficiency of the method. It is observed that the present method approximates the exact solution very well not only in the boundary layer, but also away from the layer.

In [7], author describes a Liouville-Green transform to solve a singularly perturbed two-point boundary value problem with right end boundary layer in the interval $[0,1]$. They reply Liouville-Green transform into original given problem and finds the numerical solution. Then they implemented this method on two linear examples with right end boundary layer which nicely approximate the exact solution.

In [8], author will present a new scheme for discretization of singularly perturbed boundary value problems based on finite difference methods. This method is a combination of simple upwind scheme and central difference method on a special non-uniform mesh (Shishkin mesh) for the space discretization. Numerical results show that the convergence of method is uniform with respect to singular perturbation parameter and has a higher order of convergence.

In [9], One question always strikes in every numerical analyst's mind: "Why we need higher-order methods?" Not only because they converge faster than the lower order methods but also that they provide highly accurate results in fewer number of steps. Whenever the high order accuracy is/was expected, many researchers try/tried in various different ways to design the higher-order methods. Out of the main two approaches to obtain higher-order methods; the first one is to use the methods based on the idea of acceleration of convergence whereas the other, considered in this paper, is to use the higher-order methods directly.

Here author explore the idea of deriving some higher order schemes (specially those which uses variable step sizes ) by considering the following self-adjoint singularly perturbed two point boundary value problem

$$
\begin{aligned}
& L y \equiv-\varepsilon\left(a(x) y^{\prime}\right)^{\prime}+b(x) y=f(x), \\
& x \in[0,1], y(0)=\eta_{0}, y(0)=\eta_{1}
\end{aligned}
$$

where $\eta_{0}, \eta_{1}$ are given constants and $0<\varepsilon \leq 1$. Further, $f(x), a(x)$ and $b(x)$ are sufficiently smooth functions satisfying the conditions

$$
a(x) \geq a>0, b(x) \geq b>0 .
$$

Authors systematically describe how to derive a method of higher order for the numerical solution of singularly perturbed ordinary differential equations. First they apply this idea to derive a fourth-order method for a self-adjoint singularly perturbed two point boundary value problem. This method is uniformly convergent on a piecewise uniform mesh of Shishkin type. After developing and analyzing a fourth-order method, it is explained with appropriate details, how can one obtain the methods of order higher than four which looks straightforward but has not been seen in the literature so far. Besides these, the fourth-order $\varepsilon$-uniformity in the theoretical estimate has been justified by some numerical experiments.

In [10], author present a parameter robust computational method for solving an initial value problem (IVP) for a system of first order singularly perturbed ordinary differential equations of the form

$$
\begin{aligned}
& L_{\varepsilon} u_{\varepsilon}(x)=\left\{\begin{array}{cc}
\left(L_{\varepsilon} u_{\varepsilon}\right)_{1}(x) \\
=\varepsilon D u_{\varepsilon, 1}(x)+a_{11}(x) u_{\varepsilon, 1}(x) \\
& +a_{12}(x) u_{\varepsilon, 2}(x)+\ldots \\
& +a_{1 n}(x) u_{\varepsilon, n}(x)=f_{1}(x) \\
\cdot & \\
\cdot & x \in(0, X], X>0
\end{array}\right. \\
& \left(L_{\varepsilon} u_{\varepsilon}\right)_{n}(x) \\
& =\varepsilon D u_{\varepsilon, n}(x)+a_{n 1}(x) u_{\varepsilon, 1}(x) \\
& +a_{n 2}(x) u_{\varepsilon, 2}(x)+\ldots \\
& +a_{n n}(x) u_{\varepsilon, n}(x)=f_{n}(x) \\
& u_{\varepsilon, i}(0)=u_{i}^{0} \text { for } i=1(1) n \text {, }
\end{aligned}
$$

Where $u_{\varepsilon}=\left(u_{\varepsilon, 1}, u_{\varepsilon, 2} \ldots, u_{\varepsilon, n}\right)^{T}$ and $u_{\varepsilon} \epsilon \wp^{(1)}(\Omega)$, $\bar{\Omega}=[0, X] \quad$ and $\quad D$ denotes $\frac{d}{d x}$. The functions $a_{i j}, f_{i} \in \wp^{(2)}(\bar{\Omega}), \quad i, j=1,2 \ldots \ldots, n$, satisfy the following inequalities: 
i) $a_{i i}(x)>\sum_{j=1, j \neq i}^{n}\left|a_{i j}(x)\right|, i=1(1) n$

ii) $a_{i i}(x)<0, i, j=1(1) n, i \neq j$

Both are $\forall x \in[0, X]$.

We introduce the positive number

$$
\alpha=\min \left\{\sum_{j=1}^{n} a_{1 j}, \sum_{j=1}^{n} a_{2 j} \ldots \ldots \ldots \sum_{j=1}^{n} a_{1 j},\right\},
$$

and author assume henceforth that the singular perturbation parameter $\varepsilon$ satisfies $0<\varepsilon \ll 1$. Without loss of generality we take $\mathrm{X}=1$. We introduce the notation

$$
\Omega_{0}=(0,1], \Omega=(0,1) \text { and } \bar{\Omega}=[0,1] \text {. }
$$

For a vector $\mathrm{u}$ with $\mathrm{n}$ components we use the vector norm

$$
\|u\|=\max _{i}\left|u_{i}\right|, \quad i=0(1) n
$$

and for a continuous function $\mathrm{v}$ defined on $\bar{\Omega}$ we use the continuous maximum norm

$$
\|v\|_{\bar{\Omega}}=\max _{x \in \bar{\Omega}}|v(x)|,
$$

Appropriate numerical methods for generalizations of problems of the form Eqs. (3)-(6) were presented in [11]., In this paper, it is proved that it gives essentially first order parameter uniform convergence in the maximum norm. Numerical results are presented in support of the theory.

In [12], author consider a class of singularly perturbed two-point singular boundary value problem of the form:

$$
-\varepsilon u^{\prime \prime}+\frac{k}{x} u^{\prime}(x)+g(x) u(x)=f(x), 0 \leq x \leq 1,
$$

Subject to the boundary conditions

$$
u(0)=\gamma_{0}, u(1)=\gamma_{1},
$$

where $0<\varepsilon \ll 1, f(x), \mathrm{g}(\mathrm{x})$ are bounded continuous functions in $(0,1), g(x)>0$ and $\gamma_{0}, \gamma_{1}$, are finite constants. This class of problems occurs frequently in many areas of science and engineering, for example, fluid mechanics, quantum mechanics, optimal control, chemical-reactor theory, aerodynamics, reaction diffusion process, geophysics, etc. Different numerical methods have been proposed by various authors for singularly perturbed twopoint boundary value problems [13-20]. Spline methods for solution of singularly perturbed boundary value problems are given in $[21,22,23,24]$. Mohanty et al. $[25,26,27,28]$ used various methods based on tension spline and compression spline methods on a uniform and non-uniform mesh. The use of variable mesh with finite order of accuracy for the solution of above two-point boundary value problem was studied by Stojanovic [29]. In this paper, author develop a numerical technique for a class of singularly perturbed two-point singular boundary value problems on an uniform mesh using polynomial cubic spline. The scheme derived in this paper is secondorder accurate. The resulting linear system of equations has been solved by using a tri-diagonal solver. Numerical results are provided to illustrate the proposed method and to compared with the methods in [30].
In [31], construction and analysis of non-standard finite difference methods for a class of singularly perturbed differential equations is considered. This special class consists of two types of problems: (i) those having solutions with layer behavior and (ii) those having solutions with oscillatory behavior. Since no fitted mesh method can be designed for the latter type of problems, other special treatment is necessary, which is one of the aims being attained in this paper. The main idea behind the construction of our method is motivated by the modeling rules for non-standard finite difference methods, developed by Mickens. These rules allow one to incorporate the essential physical properties of the differential equations in the numerical schemes so that they provide reliable numerical results. Note that the usual ways of constructing the fitted operator methods need the fitting factor to be incorporated in the standard finite difference scheme and then it is derived by requiring that the scheme be uniformly convergent. The method presented in this paper is fairly simple as compared to the other approaches. Several numerical examples are given to support the predicted theory.

In [32], consider a fourth-order finite-difference method for singularly perturbed one-dimensional reactiondiffusion problem of below form:

$$
\begin{aligned}
& -\varepsilon^{2} y_{\varepsilon}^{\prime \prime}(x)+b(x) y_{\varepsilon}=f(x), \\
& x \in I=[0,1], y_{\varepsilon}(0)=A, y_{\varepsilon}(1)=B,
\end{aligned}
$$

where $\varepsilon$ is the perturbation parameter, $0<\varepsilon<1$. For simplicity, it is assumed that $\mathrm{b}$ and $\mathrm{f}$ are sufficiently smooth, i.e. $b, f \in C^{\infty}(I)$ and

$$
b(x)>\beta^{2}>0, x \in I
$$

but, of course, these conditions can be somewhat relaxed, cf. [33]. The condition (10) is a standard stability condition, which implies that (9) has a unique solution $y_{\varepsilon} \in C^{\infty}(I)$.

In [34], a finite difference scheme for a class of linear singularly perturbed boundary value problems with two small parameters is considered. The problem is discretized using a Bakhvalov-type mesh. It is proved under certain conditions that this scheme is fourth-order accurate and that its error does not increase when the perturbation parameter tends to zero. Numerical examples are presented which demonstrate computationally the fourth order of the method.

In [35], semi- linear problem of below type is considered:

$$
\begin{aligned}
& -\varepsilon^{2} u^{\prime \prime}(x)+b(x, u)=0, \\
& x \in I=[0,1], u(0)=A, u(1)=B
\end{aligned}
$$

was considered under assumption

$$
b_{u}(x, u)>\beta^{2}>0, x \in I,, u \in \mathbb{R}
$$

A classical finite-difference scheme on a non-uniform mesh is used to solve (11) numerically. The discretization mesh is of Bakhvalov-type, which generalizes the idea from [36]. This enables the second order convergence uniform in $\varepsilon$. The semilinear problem of (11), (12) was 
considered in [37] with the following constraint on b in addition to (12):

$$
\begin{aligned}
& b_{u}(x, u) \leq Q(x), x \in I,, \\
& u \in \mathbb{R}, \min \left\{5 \beta^{2}-2 Q(x)\right\}>0
\end{aligned}
$$

In this paper, the method discretizes the problem (11) on a special nonequidistant mesh of Bakhvalov-type that is dense in the boundary layers. It uses a nonequidistant generalization of the fourth order three point finitedifference scheme, known as the Hermite or Numerov scheme. The uniform in $\varepsilon$ - fourth order convergence is proved. The main contribution of this paper is to construct a new uniform fourth order difference scheme for the boundary value problem (9). We extend the results given in [38] to the case of singularly perturbed problem (9) on nonequidistant mesh. Here, numerical solution of the boundary-value problem $-y^{\prime \prime}(x)+p(x) y^{\prime}+b(x) y_{\varepsilon}=f(x), \quad y(0)=A$, $y(1)=B$ on equidistant mesh was considered. Here author shall talk about finite difference schemes on special nonequidistant meshes. The reason for using these meshes is our aim to place more mesh points in the region of the boundary layers. Two-point boundary value problem are often conveniently solved with finite-difference methods that are accurate to $\vartheta\left(1 / n^{2}\right)$, where $n$ is number of subintervals. The principal attractive feature of the second-order methods is that the central difference approximations that are used ultimately lead to a tridiagonal matrix problem which may be efficiently solved using a direct elimination method. After solving the problem by this method, the truncation error is smaller than $\vartheta\left(1 / n^{2}\right)$ traditionally which have been developed by direct inclusion of higher-order differences in the approximations at each mesh point. This method produces a system of equations which is not tridiagonal and hence is more difficult to solve than the $\vartheta\left(1 / n^{2}\right)$ schemes. In this paper author present a new tridiagonal fourth order method. It is assumed that the functional coefficients of the differential equation are known analytical functions and each function is simple enough so that analytical differentiation is feasible.

In [39], a new computational method is suggested to solve the following third-order singularly perturbed boundary-value problem with a boundary layer at the left of the underlying interval in the reproducing kernel space:

$$
\left\{\begin{array}{l}
\varepsilon u^{\prime \prime \prime}(x)+a(x) u^{\prime \prime}(x)+b(x) u^{\prime}(x) \quad 0 \leq x \leq 1 \\
\quad+c(x) u(x)=f(x), \\
u^{\prime}(0)=q, u(1)=0, u^{\prime}(1)=0,
\end{array}\right.
$$

where $\quad 0<\varepsilon \ll 1, f(x) \in W_{2}^{1}[0,1], a(x), b(x), c(x)$ are sufficiently smooth function and $a(x) \geq-\alpha, \alpha>0$. In this method, first the given third-order singularly perturbed boundary-value problem is transformed into a system of two ODEs subject to suitable initial and boundary conditions and a zeroth-order asymptotic expansion for the solution of the given problem is constructed. Then the reduced terminal value problem is solved analytically in the reproducing kernel space. This method is effective and easy to implement. A numerical example is studied to demonstrate the accuracy of the present method. Results obtained by the method are found to be in good agreement with the exact solution not only in the boundary layer, but also away from the layer.

In [40], consider the following class of self-adjoint singularly perturbed two-point boundary value problems in the conservation form :

$$
\begin{aligned}
& \text { Lu } \equiv-\varepsilon\left(a(x) y^{\prime}(x)\right)^{\prime}+b(x) y(x)=g(x), \\
& \text { where } 0 \leq x \leq 1,
\end{aligned}
$$

subject to

$$
y(0)=\alpha, y(1)=\beta, \alpha, \beta \in \mathbb{R},
$$

where $\varepsilon$ is a small positive parameter and $\mathrm{a}(\mathrm{x}), \mathrm{b}(\mathrm{x})$ and $\mathrm{g}(\mathrm{x})$ are smooth functions that satisfy

$$
a(x) \geq a^{*}>0, a^{\prime}(x) \geq 0, b(x) \geq b^{*}>0 .
$$

Under these conditions, the operator L admits a maximum principle [41]. Such problems have non-smooth solutions as $\varepsilon \rightarrow 0$ with singularities related to boundary layers [42] and arise in various fields of science and engineering, for instance, fluid mechanics, quantum mechanics, optimal control, chemical-reactor theory, aerodynamics, reaction-diffusion process, geophysics etc. There are two kinds of approaches to deal with such problems: the first one is fitted mesh method which consists of choosing finer meshes in the layer region(s), and another is fitted operator method in which meshes remain uniform and the difference operator reflects the singularly perturbed nature of the differential operator. These kinds of problems using one or both of the strategies have been discussed in [43,44]. Earlier author have discussed such problems using B-spline with Shishkin mesh [45]. One of the drawbacks with the Shishkin mesh method is that, it requires a priori knowledge of the location and width of the boundary layers, therefore it motivates us to look for some adaptive methods. Recently, Lubuma and Patidar [46] have given a non-standard finite difference scheme to deal with such problems using the second approach (fitted operator).

Wavelet optimized finite difference [47] works by using an adaptive wavelet to generate irregular grids which is then exploited for the finite difference method (Lagrange finite difference in our case) and therefore it comes under fitted mesh methods. In singular perturbation problems we have shocks as boundary layers. For such kinds of problems, a solution can be smooth in most of the solution domain with a small area where the solution changes quickly. When solving such problems numerically, one would like to adjust the discretization to the solution. In terms of mesh generation (first approach), we want to have many points in an area where the solution has strong variations and few points in the area where the solution has weak variations. With a very small perturbation parameter $\varepsilon$ a large $N_{j}$ (total no. of mesh points at jth level) is required to obtain accurate solution. For a good approach of the numerical solution, at least one of the collocation points should lie in the boundary layer. For example, if the problem possesses a boundary layer of 
width $\mathrm{O}(\varepsilon)$, then on a uniform grid with $\mathrm{O}\left(N_{j}^{-1}\right)$ spacing between the points we need $N_{j}=\mathrm{O}\left(\epsilon^{-1}\right)$ which is not practically possible when $\epsilon \ll 1$ Many attempts have been done to develop numerical methods for specially designed grids that contain more points in and around the layers from time to time. Many authors developed a successful upwind central difference scheme on a piecewise uniform mesh. Wavelets have been making their presence in the field of many pure and applied areas of science and engineering [48]. Wavelets detect information at different scales and at different locations throughout the computational domain. Wavelets can provide bases in which the basis functions are constructed by dilating and translating a fixed function known as the mother wavelet (first generation wavelets). The mother wavelet can be seen as a high pass filter in the frequency domain. One of the key strength of the wavelet methods is data compression. An efficient basis is one in which a given set of data can be represented with as few basis elements as possible. Suppose we have wavelet representation of a function

$$
\sum_{k} c_{k}^{j} \varphi_{k}^{j}(x)+d_{k}^{j} \psi_{k}^{j}(x)
$$

where $\varphi_{k}^{j}(x)$ are scaling function and $\psi_{k}^{j}$ are wavelets. The coefficients of the scaling functions $c_{k}^{j}$, deal with smoother part of the function, while the wavelet coefficients $d_{k}^{j}$ contain information of the function's behavior on successive finer scales. The most common way of compressing such a representation is thresholding. We generally delete all wavelet coefficients of magnitude less than some threshold, say $\tau$. If the total number of coefficients in the original representation are $N_{j}$, we have $N_{S}$ significant coefficients left after the thresholding. Note that by thresholding a wavelet representation, we have a way to find an adaptive feature and we can also use this representation to compute function values at any point.

Author design a wavelet optimized finite difference (WOFD) scheme for solving self-adjoint singularly perturbed boundary value problems. The method is based on an interpolating wavelet transform using polynomial interpolation on dyadic grids. Small dissipation of the solution is captured significantly using an adaptive grid. The adaptive feature is performed automatically by thresholding the wavelet coefficients. Numerical examples have been solved and compared with non-standard finite difference schemes. The proposed method performs the non-standard finite difference for studying singular perturbation problems for small dissipations (very small $\varepsilon$ ) and effective grid generation. Therefore, the proposed method is better for studying the more challenging cases of singularly perturbed problems.

In [49], a new boundary value technique, which is simple to use and easy to implement, is presented for a class of linear singularly perturbed two-point boundary value problems with a boundary layer at one end (left or right) point of the underlying interval. As with other methods, the original problem is partitioned into inner and outer solution of differential equations. The method is distinguished by the following fact: the inner region problem is solved as a two-point boundary layer correction problem and the outer region problem of the differential equation is solved as initial-value problem with initial condition at end point. Some numerical experiments have been included to demonstrate the applicability of the proposed method.

In [50], a new method is presented for the following singularly perturbed problem:

$$
\left\{\begin{array}{c}
\varepsilon u^{\prime \prime}(x)+a(x) u^{\prime}(x)+b(x) u(x)=g(x), 0<x<1, \\
u(0)=0, u(1)=0,
\end{array}\right.
$$

where $0<\varepsilon \ll 1, a(x), b(x)$ and $g(x)$ are assumed to be sufficiently smooth, and $a(x) \geq \propto>0, \propto$ is a constant. Under the above assumption, (17) has a solution with a boundary layer at $x=0$. Here author only consider $u(0)=u(1)=0$, since the boundary conditions $u(0)=\propto$, $u(1)=\beta$, can be reduced to $u(0)=u(0)=u(1)=0$. In this paper, a reproducing kernel method (RKM ) is presented for solving a class of singularly perturbed boundary value problems. Firstly the original problem is reformulated as a new boundary value problem whose solution does not change rapidly via a proper transformation; then the reproducing kernel method is employed to solve the boundary value new problem. Numerical results show that the present method can provide very accurate analytical approximate solutions.

In [51], a finite difference scheme for a class of linear singularly perturbed boundary value problems with two small parameters is presented. The problem is discretized using a Bakhvalov- type mesh. It is proved under certain conditions that this scheme is fourth-order accurate and that its error does not increase when the perturbation parameter tends to zero. Numerical examples are presented which demonstrate computationally the fourth order of the method.

\section{Non-linear Singular Perturbation Boundary Value Problems}

The singularly perturbed boundary value problems (BVPs, for short) to nonlinear ordinary differential equations, which have important applications in fluid dynamics, have been studied in [52,53,54]. The existence and asymptotic estimates of solutions for singularly perturbed boundary value problem to third-order nonlinear differential equations were discussed in many papers in recent years. Many techniques arose in the studies of this kind of problem. For example, Howes [55] has considered problems of type

$$
\varepsilon^{2} y^{\prime \prime}=f(y) y^{\prime}+g(x, y), y(a)=A, y(b)=B y^{\prime}(b)=C(18)
$$

and discussed the existence and asymptotic estimates of the solutions by the method of descent. J.H.He [56] coupled the iteration method with the perturbation method to solve the well-known Blasius equation, and proved the obtained approximate analytic solutions are valid for the whole solution domain. Zhao [57] has discussed a more general class of a third-order singularly perturbed boundary value problems of the form 


$$
\varepsilon y^{\prime \prime \prime}=f\left(x, y, y^{\prime}, \varepsilon\right), y^{\prime}(0)=0, y(1)=0, y^{\prime}(1)=0
$$

and discussed the existence of solution and obtained asymptotic estimates using the theory of differential inequalities. Feckan [58] has studied high order problems and his approach was based on the nonlinear analysis involving fixed-point theory, Leray-Schauder theory, etc. $\mathrm{Du}$ et al. [59] discussed the existence, uniqueness and asymptotic estimates of solutions of the following multipoint singularly perturbed boundary value problem

$$
\begin{gathered}
\varepsilon x^{\prime \prime \prime}(t)+f\left(t, x(t), x^{\prime}(t), x^{\prime \prime}(t), \varepsilon\right)=0, \\
0 \leq t \leq 1,0<\varepsilon \ll 1, \\
x(0, \varepsilon)=0 \\
a x^{\prime}(0, \varepsilon)-b x^{\prime \prime}(0, \varepsilon)+\sum_{i=1}^{n-2} \propto_{i} x\left(\xi_{i}, \varepsilon\right)=A \\
c x^{\prime}(1, \varepsilon)+d x^{\prime \prime}(1, \varepsilon)+\sum_{i=1}^{n-2} \beta_{i} x\left(\eta_{i}, \varepsilon\right)=B .
\end{gathered}
$$

By employing of an asymptotic numerical method, Valarmathi and Ramanujam [60] have considered singularly perturbed third-order ordinary differential equations of Convection-Diffusion type. However, the boundary value problems in the above-mentioned references are all scalar and rare works are done for singular perturbation of nonlinear systems. Here, we considered few nonlinear singular perturbation problems.

In [61], we discuss singular perturbations of third-order nonlinear ordinary differential equations with full nonlinear boundary conditions. The emphasis here is that the nonlinear term depends on the first, second order derivatives and the boundary conditions are full nonlinear that is where the main novelty of this work lies. By applying the upper and lower solutions method, as well as analysis technique, the existence, uniqueness results for the singularly perturbed boundary value problem are established and asymptotic estimates of solutions is also obtained.

In [62], author study the following singularly perturbed nonlinear systems:

$$
\varepsilon^{2} x^{\prime \prime \prime}=f\left(t, x, x^{\prime}, x^{\prime \prime}\right), 0<t<1,0<\varepsilon \ll 1
$$

together with the boundary conditions

$$
\left\{\begin{array}{c}
x(0, \varepsilon)=A \\
P_{1} x^{\prime}(0, \varepsilon)-P_{2} x^{\prime \prime}(0, \varepsilon)=B \\
Q_{1} x^{\prime}(1, \varepsilon)+Q_{2} x^{\prime \prime}(1, \varepsilon)=C
\end{array}\right.
$$

where

(A) $\varepsilon$ is a small positive parameter, $\mathrm{x}, \mathrm{f}, \mathrm{A}, \mathrm{B}$ and $\mathrm{C}$ are $\mathrm{n}$ vectors, the ith component of A satisfies

$$
A_{i} \geq 0(i=1,2, \ldots \ldots \ldots n),
$$

(B) $P_{j}=\operatorname{diag}\left(p_{j 1} \ldots \ldots, p_{j n}\right), Q_{j}=\operatorname{diag}\left(q_{j 1} \ldots \ldots, q_{j n}\right)$,

$$
p_{j i} \geq 0, q_{j i} \geq 0, p_{1 i}+p_{2 i}>0 \text {, }
$$

$$
q_{1 i}+q_{2 i}>0(j=1,2 ; i=1,2, \ldots \ldots \ldots, n) .
$$

These two conditions will be assumed to hold throughout the paper.

To apply the method of descent to study BVP (22) and (23), we need to discuss the singularly perturbed second- order Volterra type integro-differential boundary value problem

$$
\begin{gathered}
\varepsilon^{2} x^{\prime \prime \prime}=f\left(t, T x, x, x^{\prime}\right), 0<t<1,0<\varepsilon \ll 1, \\
P_{1} x(0, \varepsilon)-P_{2} x^{\prime}(0, \varepsilon)=B, Q_{1} x(1, \varepsilon)+Q_{2} x^{\prime}(1, \varepsilon)=C .
\end{gathered}
$$

Author first study the problem

$$
\begin{gathered}
x^{\prime \prime}=f\left(t, T x, x, x^{\prime}\right), 0<t<1, \\
P_{1} x(0)-P_{2} x^{\prime}(0)=B, Q_{1} x(1)+Q_{2} x^{\prime}(1)=C,
\end{gathered}
$$

where

$$
[T x](t)=\left(T_{1} x_{1}, \ldots \ldots ., T_{n} x_{n}\right),
$$

$\left[T_{i} x_{i}\right](t)=A_{i}+\int_{0}^{t} x_{i}(s) d s, \quad i=1,2, \ldots \ldots .$.

This paper deals with the existence of solutions to a singularly perturbed boundary value problem for thirdorder nonlinear differential systems. The authors construct an appropriate generalized lower solution-upper solution pair, a concept that is defined in this paper, and employ the method of descent, Nagumo conditions, algebraic boundary layer functions and Volterra type integral operator to guarantee the existence of solutions of the problem. The method gives uniformly valid asymptotic estimate of the solutions. The differential systems have nonlinear dependence on all over order derivatives of the unknown.

In [63], consider the singular perturbation problem (SPP) in the quasilinear form

$$
\begin{gathered}
\varepsilon u^{\prime \prime}=f(x, u) u^{\prime}+g(x, u) \\
\text { for } x \in(a, b) \text { and } f(x, u) \neq 0, \\
u(a)=v_{a} \text { and } u(b)=v_{b},
\end{gathered}
$$

where the positive constant $\varepsilon \ll 1$ is the singular perturbation parameter, and $f(x, u)$ and $g(x, u)$ are $c^{1}$ in the domain. Assume that the right hand side $\varphi\left(x, u, u^{\prime}\right) \equiv f(x, u) u^{\prime}+g(x, u)$ of Eq. (28) satisfies a generalized Nagumo condition, Chang and Howes [64]. This simply means that every solution $u=u(x)$ satisfying $\propto(x) \leq u(x) \leq \beta(x)$ on a subinterva $J[a, b]$ has a bounded derivative, that is, there exists a constant $N=N(\propto, \beta)$ such that $\left|u^{\prime}(x)\right| \leq N$ on $J . \propto(x)$ and $\beta(x)$

are functions of $C^{2}([a, b])$. A new method is developed by detecting the boundary layer of the solution of a singular perturbation problem. On the nonboundary layer domain, the singular perturbation problem is dominated by the reduced equation which is solved with standard techniques for initial value problems. While on the boundary layer domain, it is controlled by the singular perturbation. Its numerical solution is provided with finite difference methods. This finite difference method is developed up to sixth order. The numerical error is maintained at the same level with a constant number of mesh points for a family of singular perturbation problems. Numerical experiments support the analytical results.

In [65], a method based on initial value technique is proposed for solving non-linear two-point singularly perturbed boundary value problems for second order ordinary differential equations (ODEs) with a boundary 
layer at one (either left or right) end. The original singularly perturbed boundary value problem is reduced to an initial value problem approximated by its outer solution (asymptotic approximation). The new initial value problem is solved by proposed non-linear single step explicit scheme followed the idea given in [66]. The proposed scheme has been shown to be of order two. To demonstrate the applicability of the proposed scheme several (linear and non-linear) problems have been solved. It is observed that the present scheme approximate the exact solution very well.

Consider the following nonlinear singularly perturbed boundary value problem [67]:

$$
\begin{gathered}
\left\{\begin{array}{c}
\varepsilon \frac{d z}{d t}=f(z, y, t) \\
\frac{d y}{d t}=g(z, y, t),
\end{array}\right. \\
\left\{\begin{array}{c}
a z(0, \varepsilon)+b z(1, \varepsilon)=z^{0} 0<\varepsilon \ll 1 \\
y(0, \varepsilon)=y^{0}
\end{array}\right.
\end{gathered}
$$

where $z$ and $f$ are both $M$-dimensional vectors $y$ and $g$ are both $m$-dimensional vectors and $D z$ and $D y$ are domains in $R^{M}$ and $R^{m}$ respectively. Let $a=\left(\begin{array}{cc}I_{k} & o \\ o & o\end{array}\right)$, where $I_{k}(0 \leq k \leq M)$ is a $k t h$ order identity matrix, and $b=I_{M}-a$.

In this paper, author show differentiability of solutions with respect to the given boundary value data for nonlinear singularly perturbed boundary value problems and its corresponding asymptotic expansion of small parameter. This result fills the gap caused by the solvability condition in Esipova's result so as to lay a rigorous foundation for the theory of boundary function method on which a guideline is provided as to how to apply this theory to the other forms of singularly perturbed nonlinear boundary value problems and enlarge considerably the scope of applicability and validity of the boundary function method. A third-order singularly perturbed boundary value problem arising in the theory of thin film flows is revisited to illustrate the theory of this paper. Comparing to the original result, it is found that the imposed potential condition is completely removed by the boundary function method to obtain a better result. Moreover, an improper assumption on the reduced problem has been corrected.

In [68], author present an approximate method (Initial value technique) for the numerical solution of quasilinear singularly perturbed two point boundary value problems in ordinary differential equations having a boundary layer at one end (left or right) point. It is motivated by the asymptotic behavior of singular perturbation problems. The original problem is reduced to an asymptotically equivalent first order initial value problem by approximating the zeroth order term by outer solution obtained by asymptotic expansion, and then this initial value problem is solved by an exponentially fitted finite difference scheme. Let $Y^{N}$ be the numerical approximation, $N$ be the number of mesh elements used, $y$ be the solution of the continuous problem and
$y_{\Omega}=\max _{x \epsilon \Omega}|y(x)|$ be the maximum pointwise norm, the error constant $C$ be independent of any perturbation parameters and the mesh parameter $N$. A numerical method is said to be parameter-uniform of order $p$ if

$$
\left\|y-y^{N}\right\|_{\Omega^{N}} \leq C_{p} N^{-p}, p>0
$$

where $\Omega^{N}$ is the discretization of $\Omega$ (domain of the problem), the constant $C_{p}$ is independent of any perturbation parameters and the mesh parameter $N$. In other words, the numerical approximations $Y^{N}$ converge to $y$ for all values of $\varepsilon$ in the range $0<\varepsilon \ll 1$.

It is well known that standard discretization methods for solving singular perturbation problems which are unstable and fail to give accurate results when the perturbation parameter $\varepsilon$ is small. Therefore, it is important to develop suitable numerical methods for these problems, whose accuracy does not depend on the parameter value $\varepsilon$, i.e. methods that are convergent $\varepsilon$ uniformly $[69,70]$. There are essentially two strategies to design schemes which have small truncation errors inside the layer region (s). The first approach which forms the class of fitted mesh methods consists in choosing a fine mesh in the layer region (s). The second approach is in the context of the fitted operator methods in which the mesh remains uniform and the difference schemes reflect the qualitative behavior of the solution (s) inside the layer region (s). A nice discussion using one or both of the above strategies can be found in many singular perturbation books. Some numerical examples are given to illustrate the given method. It is observed that the presented method approximates the exact solution very well for crude mesh size h.

There are a lot of physical problems that can be depicted by nonlinear ordinary differential equations (ODEs) involving some parameters. A particular interest is the solution behavior of those physical problems with one or more of the parameters being quite small. For example, for the steady-state Burgers equation [71]:

$$
\begin{gathered}
\varepsilon w^{\prime \prime}(z)-w(z) w^{\prime}(z)=0,-1<z<1, \\
w(-1)=0, w(1)=-1
\end{gathered}
$$

the parameter $\varepsilon=1 / R$ can be very small, when the Reynolds number $\mathrm{R}$ is very large.

When the boundary conditions are imposed to such type ODEs, the resulting problems are usually called the singularly perturbed boundary value problems (SPBVPs). Theoretically, the solutions of SPBVPs have to exactly satisfy the boundary conditions, but numerically this may be a quite difficult task, because a strong singularity exists within the boundary layer.

The Lie-group is a differentiable manifold, endowed with a group structure that is compatible with the underlying topology of manifold. The main purpose of Lie-group solver is providing an algorithm that can retain the orbit generated from numerical solution on the manifold which is associated with the Lie-group. The retention of Lie-group structure under discretization is vital in the recovery of qualitatively correct behavior in the minimization of numerical error [72]. 
Here, author propose a novel Lie-group shooting method for the solution of

$$
\begin{gathered}
\varepsilon u^{\prime \prime}(x)+h_{1}(x, u) u^{\prime}(x)+h_{2}(x, u)=0,0<x<1 \\
u(0)=\alpha, u(1)=\beta
\end{gathered}
$$

where $\varepsilon$ is a small parameter, $h_{1}(x, u)$ and $h_{2}(x, u)$ are given functions, $\mathrm{a}$ and $\mathrm{b}$ are given constants, and $[0,1]$ is a normalized spatial interval of our problem. The prime denotes the differential with respect to $\mathrm{x}$. This problem is a second-order nonlinear SPBVP. For the problems which are not defined in the above range, a suitable rescale of the spatial coordinate can bring them into a problem defined in the range of $x \in[0,1]$. As a demonstration, by letting $z=2 x-1$, the above steady-state Burgers equation (32) can be written as

$$
\begin{gathered}
\varepsilon u^{\prime \prime}(x)-2 u(x) u^{\prime}(x)=0,0<x<1, \\
u(0)=0, u(1)=-1,
\end{gathered}
$$

where $u(x)=w(2 x-1)$.

Now, author can transform Eqs. (34) and (35) into a mathematically equivalent first-order ODEs system:

$$
\begin{gathered}
u_{1}^{\prime}=u_{2}, \\
u_{2}^{\prime}=-\frac{1}{\epsilon}\left[h_{1}\left(x, u_{1}\right) u_{2}+h_{2}\left(x, u_{1}\right)\right], \\
u_{1}(0)=\alpha, u_{2}(1)=\beta .
\end{gathered}
$$

Eqs. (36)-(38) constitute an (u, x)-BVP, where $u(x)=\left(u_{1}(x), u_{2}(x)\right)$ denotes the system dependent variables in the $x$-domain. In order to overcome a highly singular behavior very near to the boundary as being not easy to treat by numerical method, we adopt a coordinate transformation from an $\mathrm{x}$-domain to a t-domain via a rescaling technique, which can reduce the singularity within the boundary layer. Then, we construct a Lie-group shooting method (LGSM) to search a missing initial condition through the finding of a suitable value of a parameter $r \in[0,1]$. Moreover; we can derive a closedform formula to express the initial condition in terms of $r$, which can be determined properly by an accurate matching to the right-boundary condition. Numerical examples are examined, showing that the present approach is highly efficient and accurate.

In [73], a boundary value method for solving a class of nonlinear singularly perturbed two point boundary value problems with a boundary layer at one end is proposed. Using singular perturbation analysis the method consists of solving two problems; namely, a reduced problem and a boundary layer correction problem. Author use Pade' approximation to obtain the solution of the latter problem and to satisfy the condition at infinity. Numerical examples will be given to illustrate the method.

\section{Parameterized Singular Perturbation Boundary Value Problems}

In [74], consider the following singular perturbation BVP depending on a parameter:

$$
\begin{gathered}
\varepsilon u^{\prime}(x)+f(x, y, \lambda)=0, x \in \Omega=(0, l], \\
u(0)=A, u(l)=B
\end{gathered}
$$

where $\varepsilon$ is a small positive parameter, $A$ and $B$ are given constants. The function $f(x, y, \lambda)$ is assumed to be sufficiently differentiable function in $\bar{\Omega} \times \mathbb{R}^{2}(\bar{\Omega}=\Omega \bigcup\{x=0\})$ and

$$
\begin{gathered}
0<\alpha \leq \frac{\partial f}{\partial u} \leq a^{*}<\infty, \\
0<m_{1} \leq\left|\frac{\partial f}{\partial \lambda}\right| \leq M_{1}<\infty .
\end{gathered}
$$

By a solution of (39) and (40) we mean a pair $\{u(x), \lambda\} \epsilon C^{1}(\bar{\Omega}) \times \mathbb{R}$ for which problem (39)-(40) is satisfied. For $\varepsilon \ll 1$ the function $u(x)$ has a boundary layer of thickness $O(\varepsilon)$ near $x=0$. Problems with a parameter have been considered for many years. For a discussion of existence and uniqueness results and for applications of parameterized equations see, [75-81] and references therein. In reference [82], some approximating aspects of this kind of problems has been considered. But algorithm designed for above-mentioned papers are only concerned with the regular cases (i.e., when the boundary layers are absent). The numerical analysis of singular perturbation cases has always been far from trivial because of the boundary layer behavior of the solution. Such problem undergo rapid changes within very thin layers near the boundary or inside the problem domain $[83,84,85,86,87]$. It is well known that standard numerical methods for solving such problems are unstable and fail to give accurate results when the perturbation parameter $\varepsilon$ is small. Therefore, it is important to develop suitable numerical methods to these problems, whose accuracy does not depend on the parameter value $\varepsilon$, i.e., methods that are convergence $\varepsilon$-uniformly. For the various approaches on the numerical solution of differential equations with steep, continuous solutions, author may refer to the monographs just given above. Here author analyze a finite difference scheme on a special mesh of the Bakhvalov type (a B-mesh) for the numerical solution of the BVP (39)-(40) and give a uniform first-order error estimates in a discrete maximum norm. Numerical results are presented that demonstrate the sharpness of theoretical analysis.

In [88], consider the following singularly perturbed boundary value problem with a parameter:

$$
\begin{gathered}
\varepsilon \frac{d y}{d x}+f(x, y, \lambda)=0, x \in[0,1], \\
y(0)=A, y(1)=B,
\end{gathered}
$$

where $\varepsilon$ is a small positive parameter, $A$ and $B$ are given constants. Throughout this paper, it is assumed that

$$
\begin{gathered}
f(x, y, \lambda) \epsilon C^{3}\left([0,1] \times \mathbb{R}^{2}\right), \\
\frac{\partial f}{\partial y}(x, y, \lambda) \geq a_{0}>0,(x, y, \lambda) \epsilon[0,1] \times \mathbb{R}^{2}
\end{gathered}
$$




$$
\frac{\partial f}{\partial y}(x, y, \lambda) \neq 0,(x, y, \lambda) \epsilon[0,1] \times \mathbb{R}^{2},
$$

From the solution of (41)-(42), author gets a pair $\left.\{y(x), \lambda\} \in C^{1}[0,1] \times \mathbb{R}\right), \quad$ for which (41)-(42) is satisfied. Under these assumptions, (41)-(42) has a solution $y=y(x)$, in general, displays a boundary layer of width

$$
\vartheta(\varepsilon) \text { at } x=0 \quad \text { for } \varepsilon<<1 .
$$

In this paper a novel approach is presented for solving parameterized singularly perturbed two-point boundary value problems with a boundary layer. By the boundary layer correction technique, the original problem is converted into two non-singularly perturbed problems which can be solved using traditional numerical methods, such as Runge-Kutta methods. Several non-linear problems are solved to demonstrate the applicability of the method. Numerical experiments indicate the high accuracy and the efficiency of the new method.

In [89], consider the subsequent singular perturbation boundary value problem depending on a parameter $\mathrm{k}$ :

$$
\begin{gathered}
\varepsilon u^{\prime}(t)+g(t, u, \lambda)=0, t \epsilon(0,1], \\
u(0)=A, u(1)=B,
\end{gathered}
$$

where a prime denotes derivative with respect to t, $\varepsilon$ is a small parameter, A and B are some prescribed constants. Under the assumption on $u, g$ and $\lambda$ as stated in [90], problems (43) and (44) have a unique solution pair $\{u(t), \lambda\}$. For $\varepsilon=1$ the problem considered is known as unperturbed, whereas for $\varepsilon \ll 1$ it is so-called singularly perturbed. We should remark that although the two end points enter into the problem in exactly the same manner so that the boundary layers are possible both near $t=0$ and near $t=1$, we consider the present problem only with a boundary layer of width $\mathrm{O}(\varepsilon)$ near the point $t=0$. It should also be reminded that the parameter $\mathrm{k}$ has no connection with the eigenvalue of the nonlinear differential equation under consideration, since there are two unknowns in (43) that can be determined exactly by the conditions given in (44). Here, a novel approach is presented for approximate solution of parameterized unperturbed and singularly perturbed two-point boundary value problems. The problem is first separated into a simultaneous system regarding the unknown function and the parameter, and then a methodology based on the powerful Homotopy Analysis Technique is proposed for the approximate analytic series solutions, whose convergence is guaranteed by optimally chosen convergence control parameters via square residual error. A convergence theorem is also provided. Several nonlinear problems are treated to validate the applicability, efficiency and accuracy of the method. Vicinity of the boundary layer is shown to be adequately treated and satisfactorily resolved by the method. Advantages of the method over the recently proposed conventional finitedifference method or Runga-Kutta methods are also discussed.

In [91], a singularly perturbed semi-linear boundary value problem with two-parameters is considered. The problem is solved using exponential spline on a Shishkin mesh. The convergence analysis is derived and the method is convergent independently of the perturbation parameters. Numerical results are presented which support the theoretical results.

\section{Delay Differential Equations}

A singularly perturbed delay differential equation is an ordinary differential equation in which the highest derivative is multiplied by a small parameter and involving at least one delay term. In the past, less attention had been paid for the numerical solution of singularly perturbed delay differential equations. But in recent years, there has been a growing interest in the numerical treatment of such differential equations. This is due to the versatility of such types of differential equations in the mathematical modeling of processes in various application fields, for e.g., the first exit time problem in the modeling of the activation of neuronal variability [92], in the study of bistable devices [93], evolutionary biology [94], in a variety of models for physiological processes or diseases [95], to describe the human pupil-light reflex [96] and variational problems in control theory [97] where they provide the best and in many cases the only realistic simulation of the observed phenomena. For the numerical treatment of the first order singularly perturbed delay differential equations, one can see the thesis by Tian [98]. Lange and Miura [99] gave an asymptotic approach to solve boundary value problems for the second order singularly perturbed differential difference equation with small shifts.

To approximate the solution of such boundary value problems, we consider the two cases on the basis of size of the delay (i) when the delay is of small order of the singular perturbation parameter and (ii) when the delay is of capital order of the singular perturbation parameter. In the first case, the numerical schemes proposed in [100] work nicely, but they fail in the second case, i.e., when the delay is of capital order of the singular perturbation parameter. This happens because there, we use Taylor's series to approximate the term containing the delay which is valid provided the delay is of $o(\varepsilon)$ but may lead to a bad approximation in the case when the delay is of $\mathrm{O}(\varepsilon)$. Here, we propose a generic numerical approach to solve the boundary value problem for singularly perturbed delay differential equations which works nicely in both the cases, i.e., whether the delay is of $\mathrm{O}(\varepsilon)$ or of $o(\varepsilon)$. The numerical scheme comprises a standard upwind finite difference scheme on a special type of mesh to tackle the delay argument. The restriction on the coefficient of the reaction term was relaxed. The stability and error analysis for the proposed scheme is given in both the cases, when the sign of the coefficient of the reaction term is negative or positive. An extensive amount of computation work has been carried out to demonstrate the method and to show the effect of delay on the boundary layer behavior (which is exhibited due to the presence of the singular perturbation parameter) of the solution of the problem.

In [101], a boundary value problem for second order singularly perturbed delay differential equation is considered. When the delay argument is sufficiently small to tackle the delay term, the researchers used Taylor's 
series expansion and presented an asymptotic as well as numerical approach to solve such type boundary value problem. But the existing methods in the literature fail in the case when the delay argument is bigger one because in this case, the use of Taylor's series expansion for the term containing delay may lead to a bad approximation.

Here, author present a numerical scheme for solving such type of boundary value problems, which works nicely in both the cases, i.e., when delay argument is bigger one as well as smaller one. To handle the delay argument, a special type of mesh is constructed so that the term containing delay lies on nodal points after discretization. The proposed method is analyzed for stability and convergence. To demonstrate the efficiency of the method and how the size of the delay argument and the coefficient of the delay term affect the layer behavior of the solution, several test examples are considered.

In [102], the numerical study of the boundary value problems for nonlinear singularly perturbed differentialdifference equations with small delay is discussed. Quasilinearization process is used to linearize the nonlinear differential equation. After applying the quasilinearization process to the nonlinear problem, a sequence of linearized problems is obtained. To obtain parameter-uniform convergence, a piecewise-uniform mesh is used, which is dense in the boundary layer region and coarse in the outer region. The parameter-uniform convergence analysis of the method has been discussed. The method has shown to have almost second-order parameter-uniform convergence. The effect of small shift on the boundary layer(s) has also been discussed. To demonstrate the performance of the proposed scheme, two examples have been carried out. The maximum absolute errors and uniform rates of convergence have been presented to the reader.

In [103], author study the boundary value problem (BVP) for the delay differential equation (DDE):

$$
\varepsilon u^{\prime \prime}(x)+a(x) u^{\prime}(x)+b(x) u(x-r)=f(x), x \epsilon \Omega
$$

subject to the interval and boundary conditions,

$$
u(x)=\varphi(x), x \epsilon \Omega_{0} ; u(l)=B,
$$

where $\Omega=\Omega_{1} \cup \Omega_{2}, \Omega_{1}=(0, r], \Omega_{2}=(r, l), \quad \bar{\Omega}=[0, l]$, $\Omega_{0}=[-r, 0]$ and $0<\varepsilon \leq 1$ is the perturbation parameter, $a(x) \geq \alpha>0, b(x), f(x)$ and $\varphi(x)$ are given sufficiently smooth functions satisfying certain regularity conditions to be specified and $r(l<2 r)$ is a constant delay, which is independent of $\varepsilon$ and $B$ is a given constant. For small values of $\varepsilon$ the function $\mathrm{u}(\mathrm{x})$ has a boundary layer near $x=0$. The numerical method presented here comprises a fitted difference scheme on a uniform mesh. This approach has been derived on the basis of the method of integral identities with the use of interpolating quadrature rules with the weight and remainder terms in integral form. This results in a local truncation error containing only first order derivatives of exact solution and hence facilitates examination of the convergence. In this paper, author state some important properties of the exact solution and discretize the domain by finite difference method. The error analysis for the approximate solution is presented. Uniform convergence is proved in the discrete maximum norm. Author also formulates the iterative algorithm for solving the discrete problem and present numerical results which validate the theoretical analysis computationally. The paper ends with a summary of the main conclusions. Throughout the paper, $\mathrm{C}$ denotes a generic positive constant independent of $\varepsilon$ and the mesh parameter. Some specific, fixed constants of this kind are indicated by subscripting $C$.

In [104], consider an initial value problem (IVP) for the nonlinear second-order singularly perturbed delay differential equation in the interval $\bar{I}[0, T]$ :

$$
\begin{gathered}
L u: \varepsilon u^{\prime \prime}(t)+a(t) u^{\prime}(t)+f(t, u(t), u(t-r))=0, t \epsilon I, \\
u(t)=\varphi(t), t \in I_{0} \\
u^{\prime}(0)=A / \varepsilon
\end{gathered}
$$

Where $\quad I=(0, T]=\mathcal{\cup}_{p=1}^{m} I_{p}, I_{p}=\left\{t: r_{p-1}<t \leq r_{p}\right\}$, $1 \leq p \leq m$ and $r_{s}=s r$, for $0 \leq s \leq m$ and $I_{0}=[-r, 0]$ (for simplicity we suppose that $T / r$ is integer; i.e., $T=m r \quad) . \quad 0<\varepsilon \ll 1$ is the perturbation parameter, $a(t) \geq \propto>0, f(x, u, v)$, and $\varphi(t)$ are given sufficiently smooth functions satisfying certain regularity conditions in $\bar{I}, \bar{I} \times \mathbb{R}^{2}$ and $I_{0}$ to be specified, $A$ is a constant and $r$ is a constant delay, which is independent of $\varepsilon$. Moreover

$$
\left|\frac{\partial f}{\partial u}\right| \leq b^{*} \text { and }\left|\frac{\partial f}{\partial v}\right| \leq c^{*} .
$$

In this work author present a completely exponentially fitted difference scheme on a uniform mesh for the IVP (47) and (48). The difference scheme is constructed by the method of integral identities with the use of exponential basis functions and interpolating quadrature rules with weight and remainder terms of integral form $[105,106]$. This method of approximation has the advantage that the schemes can also be effective in the case where the continuous problem is considered under certain restrictions. Some important properties of the exact solution are stated which will be needed for the analysis of the appropriate numerical solution. Here, author also presented the difference scheme and obtained uniform error estimates for the truncation terms and an appropriate solution on a uniform mesh. Numerical results are also presented.

In [107], consider a general boundary value problem for the linear second-order delay differential equation of the below form:

$$
\begin{aligned}
& \frac{\sigma^{2}}{2} u^{\prime \prime}(x)+\left(\mu_{0}-x\right) u^{\prime}(x) \\
& +\lambda_{e} u\left(x-a_{i}\right)-\left(\lambda_{e}+\lambda_{i}\right) u(x)=-1,
\end{aligned}
$$

where the values $x \equiv x_{1}$ and $x \equiv x_{2}$ corresponds to the inhibitory reversal potential and to the threshold value of membrane potential for action potential generation, respectively. Here $\sigma$ and $\mu_{0}$ are variance and drift parameters, respectively, $u$ is the expected first-exit time and the first-order derivative term $-x u^{\prime}(x)$ corresponds to exponential decay between synaptic inputs. The undifferentiated terms correspond to excitatory and 
inhibitory synaptic inputs, modeled as Poisson process with mean rates $\lambda_{e}$ and $\lambda_{i}$, respectively, and produce jumps in the membrane potential of amounts $a_{e}$ and $a_{i}$ respectively, which are small quantities and could be dependent on voltage. The boundary condition is

$$
u(x) \equiv 0, x \notin\left(x_{1}, x_{2}\right) .
$$

Here, author describe a numerical method based on fitted operator finite difference scheme for the boundary value problems for singularly perturbed delay differential equations with turning point and mixed shifts. Similar boundary value problems are encountered while simulating several real life processes for instance, first exit time problem in the modeling of neuronal variability. A rigorous analysis is carried out to obtain priori estimates on the solution and its derivatives for the considered problem. In the development of numerical methods for constructing an approximation to the solution of the problem, a special type of mesh is generated to tackle the delay term along with the turning point. Then, to develop robust numerical scheme and deal with the singularity because of the small parameter multiplying the highest order derivative term, an exponential fitting parameter is used. Several numerical examples are presented to support the theory developed in the paper.

In [108], author present a numerical study of a class of boundary value problems of singularly perturbed differential difference equations (SPDDE) which arise in computational neuroscience in particular in the modeling of neuronal variability. The mathematical modeling of the determination of the expected time for the generation of action potential in the nerve cells by random synaptic inputs in dendrites includes a general boundary-value problem for singularly perturbed differential difference equation with shifts. The problem considered in this paper exhibit turning point behavior which add to the complexity in the construction of numerical approximation to the solution of the problem as well as in obtaining theoretical estimates on the solution. Exponentially fitted finite difference scheme based on Il'in-Allen-Southwell fitting is used on a specially designed mesh. Some numerical examples are given to validate convergence and computational efficiency of the proposed numerical scheme. Effect of the shifts on the layer structure is illuminated for the considered examples.

In [109], a finite difference method is presented for singularly perturbed differential-difference equations with small shifts of mixed type (i.e., terms containing both negative shift and positive shift). Similar boundary value problems are associated with expected first exit time problems of the membrane potential in the models for the neuron. To handle the negative and positive shift terms, we construct a special type of mesh, so that the terms containing shift lie on nodal points after discretization. The proposed finite difference method works nicely when the shift parameters are smaller or bigger to perturbation parameter. An extensive amount of computational work has been carried out to demonstrate the proposed method and to show the effect of shift parameters on the boundary layer behavior or oscillatory behavior of the solution of the problem.

\section{Integral Equations}

Differential equations with integral boundary conditions constitute a very interesting and important class of problems and have been studied for many years. For a discussion of existence and uniqueness results and for applications of problems with integral boundary conditions see, below problem and the references therein:

This work [110] is concerned with singularly perturbed integral equations on the interval $I=(a, b)$. Let $\varepsilon$ be a small positive parameter. The perturbed equation is a Fredholm equation of the second kind defined as below:

$$
\epsilon u_{\epsilon}(x)+\int_{a}^{b} K(x, y) u_{\epsilon}(y) d y=f(x) .
$$

By setting $\epsilon=0$, the unperturbed equation is obtained as follows:

$$
\int_{a}^{b} K(x, y) u_{\epsilon}(y) d y=f(x)
$$

which is a Fredholm equation of the first kind. Consider kernels $K(x, y)$ defined on $[a, b] \times[a, b]$ which have the form

$$
K(x, y)=\left\{\begin{array}{l}
K_{1}(x, y), y<x, \\
K_{2}(x, y), x<y .
\end{array}\right.
$$

It is assumed that $K_{1}(x, y)$ and $K_{2}(x, y)$ are smooth on the $[a, b] \times[a, b]$. Here, several different types of kernels are considered. In particular kernels $K(x, y)$ is considered which either have a jump discontinuity along the diagonal

$$
K_{1}\left(x, x^{-}\right)-K_{2}\left(x, x^{+}\right)=a(x),
$$

Or a jump in the $n$th derivative

$$
\left.\frac{\partial^{n}}{\partial y^{n}} K_{1}(x, y)\right|_{x=y}-\left.\frac{\partial^{n}}{\partial y^{n}} K_{2}(x, y)\right|_{x=y}=a(x),
$$

where $a(x) \in C^{\infty}[a, b]$ and $a(x) \neq 0$ for $x \in[a, b]$. This is an ellipticity condition.

Suppose integral equation (52) has a kernel which satisfies the ellipticity condition (EC). Suppose reduced equation (53) has a unique solution, then the perturbed equation has a unique solution in a Sobolev space which is particularly defined for this problem. The second theorem gives the principal term in the asymptotic development of the solution and error estimates. The solution to (52) is a product of transition function and the solution to the unperturbed equation which has delta masses at the endpoints but is otherwise smooth. The transition function tends to 1 in the interior of the interval $[0,1]$ and has boundary layer terms (decaying exponentials possibly with oscillation) which may become singular at the endpoints, $x=0$ or $x=1$ as $\varepsilon \rightarrow 0$.

The purpose of this paper is to form a bridge between the work of Lange and Smith [111,112] and Eskin [113]. Lange and Smith in above reference use an additive multivariable technique to obtain solutions to singularly perturbed Fredholm equations. On the other hand, Eskin uses techniques from pseudo-differential calculus to obtain proofs of the existence and uniqueness of solutions and describe the asymptotic behavior of the solutions to a 
wide class of singularly perturbed elliptic pseudodifferential equations defined on manifolds. This work treats only one-dimensional equations on an interval and provides a rigorous, largely self-contained presentation of the main results in a simple, straight-forward manner accessible to the applied mathematician. There are several new points introduced in this paper. In particular, the index of factorization is not uniquely defined in the onedimensional case. A new criterion is found to define this index. Also variable order norms are used to describe the solution. Although such norms have appeared before, they have not been used in this context. In addition, the case of Volterra operators is not present in above given reference. The contents of the paper provides the reformulation of the problem, and contains the main theorems about the existence and uniqueness of solutions to (52) and (53) for each type of kernel and the asymptotic behavior of the solutions.

In [114], consider the following singular perturbation problem (BVP) with integral boundary condition

$$
\begin{aligned}
& \varepsilon u^{\prime}+f(t, u)=0, t \epsilon I=(0, T], T>0 \\
& u(0)=\mu u(T)+\int_{0}^{T} b(s) u(s) d s+d,
\end{aligned}
$$

where $0<\varepsilon \leq 1$ is the perturbation parameter, $\mathrm{l}$ and $\mathrm{d}$ are given constants. $b(t)$ and $f(t, u)$ are assumed to be sufficiently continuously differentiable functions in $\bar{I}=I \bigcup\{t=0\}$ and $\bar{I} \times \mathbb{R}$ respectively and moreover

$$
\frac{\partial f}{\partial u} \geq \propto>0
$$

Note that the boundary condition (57) includes periodic and initial conditions as special cases. For $\varepsilon<<1$ the function $\mathrm{u}(\mathrm{t})$ has a boundary layer of thickness $O(\varepsilon)$ near $\mathrm{t}=0$.

We consider a uniform finite difference method on Shishkin mesh for a quasilinear first order singularly perturbed boundary value problem (BVP) with integral boundary condition. Author prove that the method is first order convergent except for a logarithmic factor, in the discrete maximum norm, independently of the perturbation parameter. The parameter uniform convergence is confirmed by numerical computations.

\section{Discussion and Further Development}

In the present paper various methods for solving singularly perturbed boundary value problems are briefly discussed. It contains and analyzes huge amount of the literature related to problems of differential equations of different orders. Some problems are parameter dependent. Discretization technique is useful for solving linear or nonlinear systems of equation with a large number of unknowns. Iterative methods are commonly used to solve these systems. It should be noted that iterative method is more powerful than discretization technique. Careful must be taken when analyzing the dependence on this parameter of those constants that arise in consistency, stability and error estimates. Tailored finite point method gives high accurate results on a very coarse mesh in comparison to traditional finite element method or finite difference method. Exponential B-spline collocation method is second order uniformly convergent and gives more accuate results comparative to B-spline collocation method. Higher order cubic B-spline collocation method produces a spline function which is useful to obtain the solution at any point of the interval whereas the finite difference method gives the solution only at the selected nodal points. The extension of non-standard finite difference schemes can be applied in different type of singularly perturbed problems including turning point and non-linear problems. On the basis of above discussion, new researchers can improve the accuracy while solving the singularly perturbed boundary value problems.

\section{References}

[1] M. K. Kadalbajoo, Y.N. Reddy, Asymptotic and numerical analysis of singular perturbation problems (1989),Applied mathematics and computation 1989; 30: 223-259.

[2] M.K. Kadalbajoo and K.C. Patidar, A survey of numerical techniques for solving singularly perturbed ordinary differential equations, Applied Mathematics and Computation 130(2-3) (2002) 457-510.

[3] M. Kumar, Hradyesh Kumar Mishra, P. Singh, A recent survey on computational techniques for solving singularly perturbed boundary value problems, 2007,International Journal of Computer Mathematics 2007: 84: 1439-1463.

[4] S.A. Khuri, A. Sayfy, Self-adjoint singularly perturbed secondorder two-point boundary value problems: A patching approach, Applied Mathematical Modelling, (Article in Press) December 2013.

[5] F.Z. Geng, S.P. Qian., Reproducing kernel method for singularly perturbed turning point problems having twin boundary layers, Applied Mathematics Letters, 2013; 26: 998-1004.

[6] Essam R. El-Zahar, Approximate analytical solutions of singularly perturbed fourth order boundary value problems using differential transform method, Journal of King Saud University - Science, 2013; 25: 257-265.

[7] Hradyesh Kumar Mishra, Sonali Saini, Numerical Solution of Singularly Perturbed Two-Point Boundary Value Problem via Liouville-Green Transform, American Journal of Computational Mathematics, 2013; 3: 1-5.

[8] M.Mokarram Shahraki, S. Mohammad Hosseini, Comparison of a higher order method and the simple upwind and non-monotone methods for singularly perturbed boundary value problems, Applied Mathematics and Computation 2006;182: 460-473.

[9] Kailash C. Patidar, High order parameter uniform numerical method for singular perturbation problems, Applied Mathematics and Computation 2007; 188: 720-733.

[10] S.Hemavathi, T. Bhuvaneswari, S. Valarmathi, J.J.H. Miller, A parameter uniform numerical method for a system of singularly perturbed ordinary differential equations, Applied Mathematics and Computation 2007;191:1-11.

[11] G.I. Shishkin, Mesh approximation of singularly perturbed boundary value problems for systems of elliptic and parabolic equations, Comput. Math. Math. Phys. 1995; 35 (4): 429-446.

[12] J. Rashidinia, R. Mohammadi, M. Ghasemi Cubic spline solution of singularly perturbed boundary value problems with significant first derivatives, Applied Mathematics and Computation 2007; 190: 1762-1766.

[13] L. Abrahamson, S. Osher, Monotone difference schemes for singular perturbation problems, SIAM J. Numer. Anal. 1982;19: 979-992.

[14] P.W. Hemker, J.J.H. Miller, Numerical Analysis of Singular Perturbation Problems, Academic Press, New York, 1979.

[15] B. Kreiss, H.O. Kreiss, Numerical methods for singular perturbation problems, SIAM J. Numer. Anal.1982; 46: 138-165.

[16] J.J.H. Miller, E.O. Riordan, G.I. Shishkin, On piecewise uniform meshes for upwind and central difference operators for solving singularly perturbed problems, IMA J. Numer. Anal. 1995; 15: 8999.

[17] R.E. O’Malley, Introduction to Singular Perturbations, Academic Press, New York, 1974. 
[18] M.K. Kadalbajoo, K.C. Patidar, Numerical solution of singularly perturbed two point boundary value problems by spline in compression, Int. J. Comput. Math. 2001; 77: 263-283.

[19] R.B. Kellog, A. Tsan, Analysis of some difference approximations for a singular perturbation problem without turning points, Math. Comput. 1978; 32: 1025-1039.

[20] J. Kevorkian, J.D. Cole, Multiple Scale and Singular Perturbation Methods, Springer-Verlag, New York, 1996.

[21] R.K. Bawa, Spline based computational technique for linear singularly perturbed boundary value problems, Appl. Math. Comput. 2005; 167: 225-236.

[22] T. Aziz, A. Khan, A spline method for second order singularly perturbed boundary-value problems, J. Comput. Appl. Math. 2002; 147: 445-452.

[23] J. Rashidinia, Applications of spline to numerical solution of differential equations, M.Phil dissertation, A.M.U. India, 1990.

[24] J. Rashidinia, R. Mohammadi, R. Jalilian, The numerical solution of non-linear singular boundary value problems arising in physiology, Appl. Math. Comput. 2007; 185: 360-367.

[25] R.K. Mohanty, D.J. Evans, U. Arora, Convergent spline in tension methods for singularly perturbed two point singular boundary value problems, Int. J. Comput. Math. 2005; 82: 55-66.

[26] R.K. Mohanty, N. Jha, A class of variable mesh spline in compression method for singularly perturbed two point singular boundary value problems, Appl. Math. Comput. 2005; 168: 704716.

[27] R.K. Mohanty, N. Jha, D.J. Evans, Spline in compression method for the numerical solution of singularly perturbed two point singular boundary value problems, Int. J. Comput. Math. 2004; 81: 615-627.

[28] R.K. Mohanty, Urvashi Arora, A family of non-uniform mesh tension spline methods for singularly perturbed two-point singular boundary value problems with significant first derivatives, Appl. Math. Comput. 2006; 172 531-544.

[29] M. Stojanovic, A first order accuracy scheme on non-uniform mesh, Publ. De L'Institut. Math. 1987; 42: 155-165.

[30] M.K. Kadalbajoo, V.K. Aggarwal, Fitted mesh B-spline method for solving a class of singular singularly perturbed boundary value problems, Int. J. Comput. Math. 2005; 82: 67-76.

[31] Jean M.-S. Lubuma a, Kailash C. Patidar bNon-standard methods for singularly perturbed problems possessing oscillatory/layer solutions, Applied Mathematics and Computation 2007; 187: 1147-1160.

[32] Dragoslav Herceg, Djordje Herceg, On a fourth-order finitedifference method for singularly perturbed boundary value problems Applied Mathematics and Computation 2008; 203: 828837.

[33] R. Vulanovic', On a numerical solution of a type of singularly perturbed boundary value problem by using a special discretization mesh, Univ. Novom Sadu Zb. Rad. Prirod. Mat. Fak. Ser. Mat.1983; 13: 187-201.

[34] D. Herceg, R. Vulanovic', Some finite difference schemes for a singular perturbation problem on a nonuniform mesh, Univ. Novom Sadu Zb. Rad. Prirod. Mat. Fak. Ser. Mat. 1981; 11: 117134.

[35] R. Vulanovic', Mesh Construction for Discretization of Singularly Perturbed Boundary Value Problems, Doctoral Dissertation, Faculty of Science, Univesity of Novi Sad, 1986.

[36] N.S. Bakhvalov, Towards optimization of methods for solving boundary value problems in the presence of a boundary layer, Zh. vychisl. mat. i mat. fiz. 1969; 9: 841-859 (in Russian).

[37] D. Herceg, Uniform fourth order difference scheme for a singularly perturbation problem, Numer. Math. 1090; 56: 675-693.

[38] E.A. Bogucz, J.D.A. Walker, Fourth-order finite-difference methods for two-point boundary-value problems, IMA J. Numer. Anal. 1984; 4: 69-82.

[39] M. Cui, F. Geng, A computational method for solving third-order singularly perturbed boundary-value problems, Applied Mathematics and Computation 2008;198: 896-903.

[40] Vivek Kumar, Mani Mehra, Wavelet optimized finite difference method using interpolating wavelets for self-adjoint singularly perturbed problems, Journal of Computational and Applied Mathematics 2009; 230: 803-812.

[41] M.H. Protter, H.F. Weinberger, Maximum Principles in Differential Equations, Prantice-Hall, Inc., Englewood Cliffs, NJ, 1967.

[42] H.G. Roos, M. Stynes, L. Tobiska, Numerical Methods for Singularly Perturbed Differential Equations, Springer, 1996.
[43] J.J.H. Miller, E. O'Riordan, I.G. Shishkin, Fitted Numerical Methods for Singular Perturbation Problems, World Scientific, 1996.

[44] P.A. Farrell, A.F. Hegarty, J.J.H. Miller, E. O'Riordan, I.G. Shishkin, Robust Computational Techniques for Boundary Layers, Chapman and Hall, CRC, 2000.

[45] M.K. Kadalbajoo, V.K. Aggarwal, Fitted mesh B-spline collocation method for solving self adjoint singularly perturbed boundary value problems, Appl. Math. Comput.2005; 161 (3): 973-987.

[46] J.M.S. Lubuma, K.C. Patidar, Uniformly convergent non-standard finite difference methods for self-adjoint singular perturbation problems, J. Comput. Appl. Math. 2006; 191: 228-238.

[47] L. Jameson, A wavelet-optimized, very high order adaptive grid and numerical method, SIAM J. Sci. Comput. 1998; 19: $1980-$ 2013.

[48] V. Kumar, M. Mehra, Cubic spline adaptive wavelet scheme to solve singularly perturbed reaction diffusion problems, Int. J. Wavelets Multiresoult. Inf. Process. 2007; 5 (2): 317-331.

[49] Manoj Kumar, Hradyesh Kumar Mishra, Peetam Singh, A boundary value approach for a class of linear singularly perturbed boundary value problems, Advances in Engineering Software 2009; 40: 298-304.

[50] Fazhan Geng, A novel method for solving a class of singularly perturbed boundary value problems based on reproducing kernel method. Applied Mathematics and Computation 2011; 218: 42114215.

[51] Djordje Herceg, Fourth-order finite-difference method for boundary value problems with two small parameters. Applied Mathematics and Computation 2011; 218: 616-627.

[52] N. Levinson, A boundary value problem for a singularly perturbed differential equation, Duke Math. J. 1958; 25: 331-343.

[53] D.M. Goecke, Third-order differential inequalities and singular perturbations, Doctoral Dissertation, Univ. Oklahoma, Norman, 1979.

[54] F.A. Howes, Differential inequalities of higher order and the asymptotic solution of nonlinear boundary value problems, SIAM J. Appl. Math. 1982; 13: 61-80.

[55] F.A. Howes, The asymptotic solution of a class of third-order boundary value problem arising in the theory of thin film flow, SIAM J. Appl. Math.1983; 43: 993-1004.

[56] Jihuan He, A simple perturbation approach to Blasius equation, Appl. Math. Comput. 2003; 140: 217-222.

[57] Weili Zhao, Singular perturbations of boundary value problems for a class of third-order nonlinear ordinary differential equations, J. Differen. Equat. 1990; 88: 265-278.

[58] M. Feckon, Singularly perturbed high order boundary value problems, J. Differen. Equat. 1994; 11: 79-102.

[59] Zengji Du, Weigao Ge, Mingru Zhou, Singular perturbations for third-order nonlinear multi-point boundary value problem, J. Differen. Equat. 2005; 218 (1): 69-90.

[60] S. Valarmathi, N. Ramanujam, An asymptotic numerical method for singular perturbed third-order differential equations of convection-diffusion type, Comput. Math. Appl. 2002; 44: 693710.

[61] Xiaojie Lin, Singular perturbations of third-order nonlinear differential equations with full nonlinear boundary conditions, Applied Mathematics and Computation, 2013; 224:88-95.

[62] Zengji Du, Singularly perturbed third-order boundary value problem for nonlinear systems,applied Mathematics and Computation 2007;189 : 869-877.

[63] Tzu-Chu Lin, David H. Schultz, Weiqun Zhang, Numerical solutions of linear and nonlinear singular perturbation problems, Computers and Mathematics with Applications 2008; 55: 25742592.

[64] R.E. O’Malley Jr., Introduction to Singular Perturbations, Academic Press, New York, 1974.

[65] Mohan K. Kadalbajoo, Devendra Kumar, A non-linear single step explicit scheme for non-linear two-point singularly perturbed boundary value problems via initial value technique. Applied Mathematics and Computation 2008; 202: 738-746.

[66] F.D. Van Niekerk, Non-linear one-step methods for initial value problems, Comput. Math. Appl. 1987; 13: 367-371.

[67] Zhiming Wang, Wuzhong Lin, Gexia Wang, Differentiability and its asymptotic analysis for nonlinear singularly perturbed boundary value problem Nonlinear Analysis 2008; 69: 2236-2250. 
[68] Mohan K. Kadalbajoo, Devendra Kumar, Initial value technique for singularly perturbed two point boundary value problems using an exponentially fitted finite difference scheme, Computers and Mathematics with Applications 2009; 57: 1147-1156.

[69] H.G. Roos, A second order monotone upwind scheme, Computing 1986; 36: 57-67.

[70] E.P. Doolan, J.J.H. Miller, W.H.A. Schilders, Uniform Numerical Methods for Problems with Initial and Boundary Layers, Boole Press, Dublin, 1980.

[71] Chein-Shan Liu, The Lie-group shooting method for solving nonlinear singularly perturbed boundary value problems, Commun Nonlinear Sci Numer Simulat 2012;17:1506-1521.

[72] R.E. O'Mally, Singular Perturbation Methods for Ordinary Differential Equations, Springer, New York, 1991.

[73] Basem S. Attili, Numerical treatment of singularly perturbed two point boundary value problems exhibiting boundary layers Commun Nonlinear Sci Numer Simulat 2011; 16: 3504-3511.

[74] G.M. Amiraliyev, Mustafa Kudu, Hakki Duru, Uniform difference method for a parameterized singular perturbation problem. Applied Mathematics and Computation2006; 175: 89-100.

[75] K. Zawischa, Uber die Differentialgleichung deren Losungskurve durch zwei gegebene Punkte hindurchgehen soll, Monatsh. Math. Phys. 1930; 37: 103-124.

[76] T. Pomentale, A constructive theorem of existence and uniqueness for problem $y 0=f(x, y, k), y(a)=a, y(b)=b, Z$. Angew. Math. Mech. 1976; 56: 387-388.

[77] M. Feckan, Parametrized singularly perturbed boundary value problems, J. Math. Anal. Appl.1994; 188:426-435.

[78] T. Jankowski, Monotone iterations for differential problems, Math. Notes, Miscolc 2001; 2: 31-38.

[79] T. Jankowski, V. Lakshmikantham, Monotone iterations for differential equations with a parameter, J. Appl. Math. Stoch. Anal. 1997; 10: 273-278.

[80] M. Ronto, T. Csikos-Marinets, On the investigation of some nonlinear boundary value problems with parameters, Math. Notes, Miscolc 2000; 1: 157-166.

[81] S. Stanek, Nonlinear boundary value problem for second order differential equations depending on a parameter, Math. Slovaca 1997; 47: 439-449.

[82] A. Gulle, H. Duru, Convergence of the iterative process to the solution of the boundary problem with the parameter, Trans. Acad. Sci. Azerb., Ser. Phys. Tech. Math. Sci. 1998; 18: 34-40.

[83] A.H. Nayfeh, Introduction to Perturbation Techniques, Wiley, New York, 1993

[84] E.R. Doolan, J.J.H. Miller, W.H.A. Schilders, Uniform Numerical Methods for Problems with Initial and Boundary Layers, Boole, Press, Dublin, 1980.

[85] G.M. Amiraliyev, Difference method for the solution one problem of the theory dispersive waves, USSR Diff. Equat. 1990; 26: 21462154.

[86] G.M. Amiraliyev, H. Duru, A uniformly convergent finite difference method for a singularly perturbed initial value problem, Appl. Math. Mech. (English Edition) 1999; 20: 379-387.

[87] G.M. Amiraliyev, H. Duru, A uniformly convergent difference method for the periodical boundary value problem, Comput. Math. Appl.2003; 46:695-703.

[88] Feng Xie, JianWang, Weijiang Zhang, Ming He, A novel method for a class of parameterized singularly perturbed boundary value problems Journal of Computational and Applied Mathematics 2008; 213: 258-267.

[89] M.Turkyilmazoglu, Analytic approximate solutions of parameterized unperturbed and singularly perturbed boundary value problems. Applied Mathematical Modelling 2011, 35: 38793886.

[90] G.M. Amiraliyev, H. Duru, A note on a parameterized singular perturbation problem, J. Comput. Appl. Math. 2005; 182: 233-242.

[91] W.K. Zahra, Ashraf M. El Mhlawy, Numerical solution of twoparameter singularly perturbed boundary value problems via exponential spline, Journal of King Saud University - Science, 2013; 25: 201-208.

[92] C.G. Lange, R.M. Miura, Singular perturbation analysis of boundary-value problems for differential-difference equations. $\mathrm{v}$. small shifts with layer behavior, SIAM J. Appl. Math. 1994; 54: 249-272.

[93] M.W. Derstine, F.A.H.H.M. Gibbs, D.L. Kaplan, Bifurcation gap in a hybrid optical system, Phys. Rev. A 1982;26: 3720-3722.
[94] M. Wazewska-Czyzewska, A. Lasota, Mathematical models of the red cell system, Mat. Stos. 1976; 6: 25-40.

[95] M.C. Mackey, G.L. Oscillations and chaos in physiological control systems, Science, 1977; 197: 287-289.

[96] A. Longtin, J. Milton, Complex oscillations in the human pupil light reflex with mixed and delayed feedback, Math. Biosci. 1988; 90: 183-199.

[97] V.Y. Glizer, Asymptotic analysis and solution of a finite-horizon H1 control problem for singularly-perturbed linear systems with small state delay, J. Optim. Theory Appl. 2003; 117: 295-325.

[98] H. Tian, Numerical treatment of singularly perturbed delay differential equations, $\mathrm{PhD}$ thesis, University of Manchester, 2000.

[99] C.G. Lange, R.M. Miura, Singular perturbation analysis of boundary-value problems for differential-difference equations. vi. Small shifts with rapid oscillations, SIAM J. Appl. Math.1994; 54: 273-283.

[100] M.K. Kadalbajoo, K.K. Sharma, Numerical analysis of singularly perturbed delay differential equations with layer behavior, Appl. Math. Comput. 2004; 157: 11-28.

[101] Mohan K. Kadalbajoo, Kapil K. Sharma,, A numerical method based on finite difference for boundary value problems for singularly perturbed delay differential equations, Applied Mathematics and Computation 2008;197: 692-707.

[102] Mohan K. Kadalbajoo, Devendra Kumar, A computational method for singularly perturbed nonlinear differential-difference equations with small shift. Applied Mathematical Modelling 2010; 34: 25842596.

[103] Gabil M. Amiraliyev, Erkan Cimen, Numerical method for a singularly perturbed convection-diffusion problem with delay Applied Mathematics and Computation 2010; 216: 2351-2359.

[104] I.G. Amiraliyeva, F. Erdogan, G.M. Amiraliyev, A uniform numerical method for dealing with a singularly perturbed delay initial value problem Applied Mathematics Letters 2010; 23: 1221-1225.

[105] G.M. Amiraliyev, Y.D. Mamedov, Difference schemes on the uniform mesh for singularly perturbed pseudo-parabolic equations, Tr. J. Math. 1995; 19: 207-222.

[106] G.M. Amiraliyev, H. Duru, A uniformly convergent finite difference method for a initial value problem, Appl. Math. Mech.1999; 20 (4):363-370.

[107] Pratima Rai, Kapil K. Sharma, Numerical analysis of singularly perturbed delay differential turning point problem. Applied Mathematics and Computation 2011; 218: 3483-3498.

[108] Pratima Rai, Kapil K. Sharma, Numerical study of singularly perturbed differential-difference equation arising in the modeling of neuronal variability, International Journal of computer mathematics with applications, 2012; 63 (1): 118-132.

[109] R. Nageshwar Rao, P. Pramod Chakravarthy, A finite difference method for singularly perturbed differential-difference equations arising from a model of neuronal variability, Journal of Taibah University for Science,2013; 73: 128-136.

[110] Carol Shubin, Singularly perturbed integral equations, J. Math. Anal. Appl.2006; 313: 234-250.

[111] C. Lange, D. Smith, Singular perturbation analysis of integral equations, Stud. Appl. Math. 1988; 79: 1-63.

[112] C. Lange, D. Smith, Singular perturbation analysis of integral equations, Part II, Stud. Appl. Math. 1993; 90: 1-74.

[113] G. Eskin, Asymptotics of solutions of elliptic pseudo-differential equations with a small parameter, Dokl. Akad. Nauk USSR 211 (1973) 547-550. English translation: Soviet Math. Dokl. 1973; 14: 1080-1083.

[114] G.M. Amiraliyev, I.G. Amiraliyeva, Mustafa Kudu, A numerical treatment for singularly perturbed differential equations with integral boundary condition Applied Mathematics and Computation 2007; 185: 574-582. 